\title{
ARTICLE
}

\section{Differentiator Application in Altitude Control for an Indoor Blimp Robot}

\author{
Yue Wang $^{\mathrm{ab}}$, Gang Zheng ${ }^{\mathrm{ab}}$, Denis Efimov ${ }^{\mathrm{abc}}$, Wilfrid Perruquetti ${ }^{\mathrm{ab}}$ \\ ${ }^{a}$ CRIStAL (CNRS UMR 9189), Ecole Centrale de Lille, 59650 Villeneuve d'Ascq,

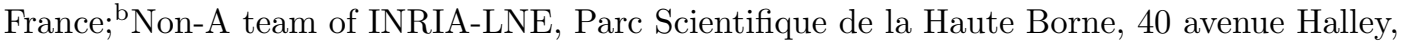 \\ Building A Park Plaza, 59650 Villeneuve d'Ascq, France; ${ }^{c}$ Department of Control Systems \\ and Informatics, ITMO University, Saint Petersburg, Russia
}

\author{
ARTICLE HISTORY \\ Compiled October 5, 2018
}

\begin{abstract}
This paper presents design of altitude controller with disturbance compensation for an indoor blimp robot and its realisation. Due to hardware restrictions, the altitude control behaviour of blimp is modelled as a switched system with a constant time-delay complemented with uncertain bounded disturbances. In order to achieve state estimation, four differentiators are applied and compared, then HOMD (homogeneous finite-time) differentiator is chosen as an observer for vertical velocity and switching signal estimation. Next, a predictor-based controller is conceived, and in order to compensate the perturbation, the method for disturbance evaluation is designed still with the help of HOMD differentiator. Control scheme is implemented by Matlab Simulink, and finally, the performance of blimp altitude controller is verified in experiments.
\end{abstract}

\section{KEYWORDS}

Differentiator, Flying robots; Output feedback control; Control of switched system

\section{Introduction}

Robotics is a quickly developing area of science and technology nowadays. Frequently, robots are developed for replacing humans in dangerous operating conditions or for optimisation of manufacturing expenses. According to their operating environment, robots can be classified into two types: indoor or outdoor, since depending on that they have different restrictions on dimensions, noise level, actuators and sensors used. Among the flying robots it is worth to mention airships or blimps which are lighterthan-air (LTA) aircrafts, for their long endurance in air, high payload-to-weight ratio, and low noise level features, compare with fixed-wing aircrafts and rotor-wing aircrafts (Li, Nahon, \& Sharf, 2011).

In the present work, a small blimp robot for indoor operation is considered. This robot has to possess a good autonomy operation time and demonstrate a low noise production level. The blimp can be used to do indoor long-term monitoring, or unknown dangerous environment exploring etc. In order to accomplish those complex goals, it has to be well controlled under different scenarios, and stabilising the blimp 
at a desired altitude is the basic requirement. Thus, the problem of robust altitude stabilisation is considered in this work. To this end, based on real data obtained for the blimp platform available in our laboratory, a switched time-delay model of altitude dynamics is identified. Different differentiators are applied and compared for state and disturbance estimation purposes. Next, predictor-based control is designed. The obtained solutions are experimentally verified.

This paper is organised as follows. In the next section, some related works are introduced and discussed. Structure of our blimp system is presented in section 3 , the altitude model and its parameter identification are also discussed in this section. In section 4, in order to realise state observation, four differentiators are briefly reviewed and compared to select the most appropriate one. A controller design based on predictor is illustrated; moreover, the method to estimate and compensate disturbance of system is conceived. Afterwards, we focus on the real blimp, and using a motion capture system to enhance the control scheme, experiment results are presented in section 5. Finally, conclusion comes in section 6 .

\section{Related Works}

Researchers have put increasing attention on autonomous blimp robots over the last few years; they use airships as experiment platforms to study information acquisition, robot control and navigation algorithms.

Many of these blimps are of large scale, which can only be tested outdoor. They normally have a payload of several kilograms, which allow them to carry plenty of high-precision sensors to do experiments. For instance, Hygounenc, Jung, Soueres, and Lacroix (2004) used blimp in their terrain mapping research, achieved positioning of blimp in the three-dimensional space with a centimetre accuracy. Kantor, Wettergreen, Ostrowski, and Singh (2001) discussed the use of solar energy as a renewable source of power for airships, which they used for environmental data sampling and monitoring. Rao, Gong, Luo, and Xie (2005) proposed a fuzzy logic controller which was based on the dynamics of the vehicle for heading angle and altitude control; their robot realised $3 \mathrm{D}$ path tracking with acceptable error.

Furthermore, other researchers made various studies of blimps in indoor environments, such as localisation of robot, obstacle avoidance algorithm, trajectory planning and path tracking control. Wyeth and Barron (1998) used landmark navigation system to navigate their blimp. Fukao, Fujitani, and Kanade (2003) installed a camera on airship for surveillance system and illustrated an image-based tracking control for an indoor blimp. Green, Sevcik, and Oh (2005) also used camera on blimp to realise obstacle avoidance function. Zufferey, Guanella, Beyeler, and Floreano (2006) used neuronal controllers in simulation to map visual input into motor commands to accelerate the movement of flying robot while avoiding collisions. Ko, Klein, Fox, and Haehnel (2007) combined their Gaussian processes enhanced model to reinforcement learning and designed a controller for blimp's yaw and yaw rate control. Rottmann, Zitterell, Burgard, Reindl, and Scholl (2007) also used reinforcement learning to design a controller which can be used to control blimp's altitude without knowing the specific dynamics of the system or other environmental parameters. López Fernández, González, Sanz, and Burgard (2009) designed PID controller together with fuzzy logic controller to realise airship's altitude control; the ultrasonic sensor information is used to accomplish collision avoidance.

As we can see from the related works, some of them did not propose models for 
indoor blimp robot, while the others built complex models, which need lots of experimental data to identify the parameters but there are still disturbances from the environment which cannot be modelled. Since an indoor blimp robot is considered in this work, it has to be sufficiently small, and its payload is limited. This limits possible quantity of sensors mounted on the robot and computational complexity of the control and estimation algorithms. That is why we intend to use a simple model for blimp's altitude control task, and design a controller which can estimate and compensate the disturbances in real time (Guerra et al. 2015), so that the accuracy of control is assured while the complexity is reduced to a minimum. Our developed modelling, estimation and control algorithms are based on real data measurements and experimental validation performed on real platform, which is described in the next section.

\section{Blimp System}

We will consider a blimp robot for indoor operation, with a Mylar balloon of $110 \mathrm{~cm}$ length and $70 \mathrm{~cm}$ of maximum diameter. Helium is used to inflate the balloon so it has a payload of about 160 grams. Therefore, only low-weight sensors and actuators can be integrated in an embedded micro-system, which means the measurement of on-board sensors cannot be very accurate. On the latest control board, the micro-controller used is STM32, which controls four mini motors - two work in the horizontal plane and the other two work in the vertical direction. The wireless communication between control board on blimp and ground PC is realised by a pair of XBee modules. In order to improve the altitude measurement accuracy, the high-precision position and pose capturing system OptiTrack is applied to enhance the blimp system. It uses infrared waves to capture the markers mounted on blimp control board, and solves the blimp position with a precision of $1 \mathrm{~mm}$.

\section{1. $\quad$ Altitude Control Model}

In order to develop the control of blimp, the 6 DOF kinematic model is considered. Fig. (1) shows the coordinate and symbols of blimp variables (Rao et al., 2005).

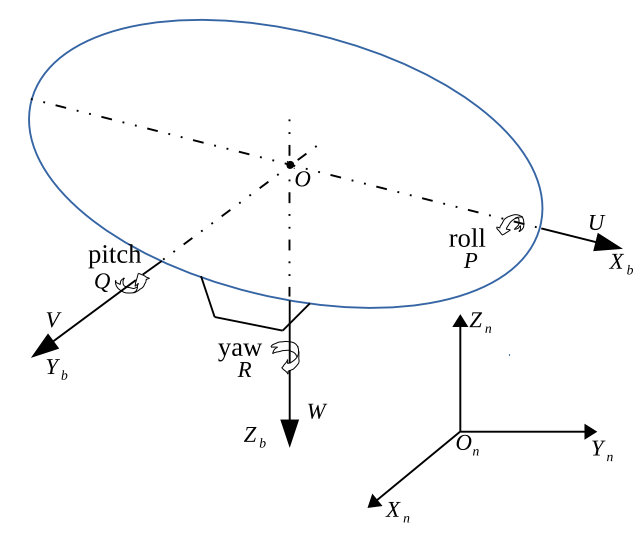

Figure 1. Coordinate and symbols of blimp

The motion of blimp is referenced to a body-fixed orthogonal frame $R_{b}\left(O, X_{b}, Y_{b}, Z_{b}\right)$; blimp linear and angular velocities are given by $\xi=$ 
$\left[\begin{array}{llllll}U & V & W & P & Q & R\end{array}\right]^{\mathrm{T}}$. The position and orientation of frame $R_{b}$ with respect to navigation frame $R_{n}\left(O_{n}, X_{n}, Y_{n}, Z_{n}\right)$ is denoted as $\eta=\left[\begin{array}{llllll}x & y & z & \phi & \theta & \psi\end{array}\right]^{\mathrm{T}}$. The kinematic model between frame $R_{b}$ and $R_{n}$ is given by

$$
\dot{\eta}=J(\eta) \xi
$$

where $J(\eta)$ is the transition matrix from $R_{b}$ to $R_{n}$.

Following investigation of Hygounenc et al. (2004) that the state parameters of airship dynamics in lateral and longitudinal motions are weakly dependent, the dynamics of blimp can be split into two subsystems: the one within longitudinal plane $\left(\eta_{\text {long }}=\left[\begin{array}{lll}x & z & \theta\end{array}\right]^{\mathrm{T}}, \xi_{\text {long }}=\left[\begin{array}{lll}U & W & Q\end{array}\right]^{\mathrm{T}}\right)$, and the one within lateral plane $\left(\eta_{\text {lat }}=\left[\begin{array}{lll}y & \phi & \psi\end{array}\right]^{\mathrm{T}}, \xi_{\text {lat }}=\left[\begin{array}{lll}V & P & R\end{array}\right]^{\mathrm{T}}\right)$. For blimp dynamics in longitudinal plane, it is imperative to stabilise the airship at a desired altitude since it is the fundamental demand of blimps to perform complex tasks. In fact, altitude control is already a complex task as a blimp is really sensitive to environment changes and its behaviour is easily influenced by many factors like the payload change, battery level, air flow disturbances, and temperature.

First, in this work, we intend to model the altitude control behaviour of blimp and identify the parameters. To this end, the dynamical equation in the vertical axis is considered. The blimp is subjected to its gravity, buoyancy, the propulsive force by its vertical motor, and the air resistance when it moves. So for the dynamical equation along the vertical axis, based on Newtonian principles, there is

$$
m \ddot{z}=F_{p}+F_{b}-G-F_{r}
$$

where $z$ is the altitude of blimp to the reference plane, $F_{p}, F_{b}, G, F_{r}$ represent propulsive force, buoyancy, gravity, and air resistance, respectively.

The equation is simplified with the following assumptions:

- the buoyancy and gravity of blimp do not change wherever it is in the testing environment;

- the buoyancy and gravity of blimp are approximately equal;

- the air resistance is proportional to blimp velocity (the blimp has low speed);

- the propulsive force is proportional to square of motor rotation speed, which is modelled as input;

- the delay between emission of control signal and the rotation speed change of the motor is denoted as $\tau$.

So, Equation (2) is simplified as

$$
\ddot{z}(t)=a \dot{z}(t)+b u(t-\tau)+c
$$

where $c$ represents the resultant force of buoyancy and gravity of blimp, $a, b$, and $\tau$ are the parameters which have to be estimated to validate the model. 


\subsection{Parameter Identification}

In order to identify the model parameters, the input $u$ for vertical motor is set as a step signal, and blimp altitude is measured using the OptiTrack system with a rate of $100 \mathrm{~Hz}$. In our tests there is a time-varying delay measured from 0.2 to $0.4 \mathrm{~s}$ in blimp control loop due to the wireless transmission and motor settling time; therefore, the delay has to be manually measured in different tests.

After the delay $\tau$ is measured, the parameters $a, b$ can be identified. For a step input with gain $N$, assume at the beginning, blimp is static, i.e. $\dot{z}(0)=0, c=0$, using Laplace transform at the differential equation (3), we get

$$
Z(s)=\frac{N b}{s^{2}(s-a)} e^{-\tau s}+\frac{z(0)}{s}
$$

Using inverse Laplace transform, we get

$$
z(t)=z(0)+N\left(\frac{b}{a^{2}} e^{a(t-\tau)}-\frac{b}{a^{2}}-\frac{b}{a}(t-\tau)\right)
$$

Then, the Levenberg-Marquardt nonlinear least squares algorithm is used to estimate parameters in the given function (Seber \& Wild, 2003).

It is worth to mention that the motor rotation speed is not the same in practice when it rotates in forward/backward direction with the same input value due to motor defect. Besides, the air resistance coefficient differs when the blimp moves upward and downward on account of the installation of control board at bottom of balloon.

Therefore, step input tests with positive and negative final value are made to separately identify parameters $a$ and $b$; they have to be switched according to blimp state (direction of movement) and motor command. See Table 1 for the parameter identification result. Note that $a$ is related to the air-resistant coefficient, and $b$ is related to the propulsive force.

Table 1. Parameter identification result

\begin{tabular}{lll}
\hline Case & \multicolumn{1}{c}{$\dot{z} \geq 0$} & \multicolumn{1}{c}{$\dot{z}<0$} \\
\hline \multirow{2}{*}{$u \geq 0$} & $\begin{array}{l}a=-0.035 \\
b=0.0628\end{array}$ & $\begin{array}{l}a=-0.0366 \\
b=0.0628\end{array}$ \\
\hline \multirow{2}{*}{$u<0$} & $a=-0.035$ & $a=-0.0366$ \\
& $b=0.0424$ & $b=0.0424$ \\
\hline
\end{tabular}

\subsection{System Description}

The parameter identified in Section 3.2 can be used to establish a nominal model for blimp altitude control system. Then we intend to use a disturbance term to represent the errors between nominal model and real one, which includes the errors caused by:

- Nominal model parameter identification inaccuracy;

- Difference between blimp buoyancy force and gravity;

- Helium leak of the balloon;

- Airflow perturbation to balloon;

- Variation of time-delay in the control loop; 
- Temperature change in testing environment;

- Other environmental disturbances which are impossible to be accurately modelled.

Therefore, the blimp altitude control system studied in this paper is considered as a switched system with a constant time-delay complemented with uncertain bounded disturbances (Guerra et al., 2015). Blimp altitude $z$ and velocity in vertical axis $\dot{z}$ are chosen as the state vector $X=\left[\begin{array}{ll}z & \dot{z}\end{array}\right]^{\mathrm{T}}$; the system can be written in state-space form as the combination of nominal model and real-time estimated disturbance term:

$$
\left\{\begin{aligned}
\dot{X}(t) & =A_{\sigma} X(t)+B_{\sigma} u\left(t-\tau_{\text {nom }}\right)+B_{\sigma} d(t) \\
y(t) & =C X(t)
\end{aligned}\right.
$$

where $A_{\sigma}=\left[\begin{array}{cc}0 & 1 \\ 0 & a_{\sigma}\end{array}\right], B_{\sigma}=\left[\begin{array}{c}0 \\ b_{\sigma}\end{array}\right]$, with parameters identified for nominal model, $C=\left[\begin{array}{ll}1 & 0\end{array}\right], \tau_{\text {nom }}$ is the nominal delay in control loop, estimated in tests as $\tau_{\text {nom }}=$ $0.3 \mathrm{~s}, d(t)$ is the disturbance term which is estimated online. In order to facilitate calculation, a coefficient $B_{\sigma}$ is added to $d(t), B_{\sigma} d(t)$ is assumed to be small, bounded and smooth.

Switching signal:

$$
\sigma= \begin{cases}1, & u \geq 0 \text { and } \dot{z} \geq 0 \\ 2, & u \geq 0 \text { and } \dot{z}<0 \\ 3, & u<0 \text { and } \dot{z} \geq 0 \\ 4, & u<0 \text { and } \dot{z}<0\end{cases}
$$

From the results in Section 3.2 , there is (Table 1)

$$
\begin{aligned}
& a_{1}=a_{3}=-0.035 \\
& a_{2}=a_{4}=-0.0366 \\
& b_{1}=b_{2}=0.0628 \\
& b_{3}=b_{4}=0.0424
\end{aligned}
$$

\section{Altitude Controller Design}

In order to solve the altitude stabilisation problem for system (6), an output feedback should be designed. For this purpose, in this work, the following steps are proposed:

1) Design an observer which can estimate state and switching signal of time-delay switched system (6).

2) Design a controller which stabilises the system in the nominal disturbance-free case.

3) Modify or develop the control algorithm to compensate the disturbance. 


\subsection{Differentiator Observer Design}

As the sensors in system can only measure blimp altitude, in order to use state feedback to design the closed-loop system, both components of the state $z$ and $\dot{z}$ have to be known. Moreover, the switching signal $\sigma$ has to be also estimated to give commutation information about the system. As $\sigma$ is determined by the velocity $\dot{z}$ and command $u$ (see criteria (7)), $\dot{z}$ has to be calculated.

The system studied is a switched system with a constant time-delay complemented with uncertain bounded disturbances as described in Section 3.3. A Luenberger observer can be used to estimate all the states, but the switching feature of system increases the complexity of observer design and the precision of estimation result cannot be guaranteed. Considering the fact that required state is the first-order derivative of measurement, a differentiator is more suitable for the state estimation task.

For this purpose four different differentiators are considered: High-Gain, HOSM (high-order sliding mode), HOMD (homogeneous finite-time), and algebraic-based (ALIEN) differentiator. First, a brief overview of these differentiators is given, and then, tests are made to compare their performances in our application scenario.

\subsubsection{ALIEN Differentiator}

The algebraic differentiator is proposed in the paper of Sira-Ramirez and Fliess (2006). Consider a signal $x(t)$ which is assumed to be analytic on a finite-time interval. The truncated Taylor expansion of $x(t)$ is

$$
x_{N}(t)=\sum_{k=0}^{N} x^{(k)}(0) \frac{t^{k}}{k !}
$$

The Laplace transform of $(9)$ is

$$
X_{N}(s)=\sum_{k=0}^{N} \frac{x^{(k)}(0)}{s^{k+1}}
$$

Multiplying both sides of 100 by $\frac{d^{\nu}}{d s^{\nu}} s^{N}, \nu=0,1, \ldots, N$, we get

$$
\frac{d^{\nu} s^{N}}{d s^{\nu}} X_{N}(s)=\frac{d^{\nu}}{d s^{\nu}}\left(\sum_{k=0}^{N} x^{(k)}(0) s^{N-k-1}\right)
$$

According to Fliess and Sira-Ramírez (2003), (11) is independent of unknown initial conditions and $x(0), \ldots, x^{(k)}(0)$ are linearly identifiable. Therefore, the $k$-th-order derivative $x^{(k)}(0)$ can be estimated by taking inverse Laplace transform of (11) over a time window $T$.

The ALIEN differentiator has the following form according to Mboup, Join, and Fliess (2007):

$$
\left\{\begin{array}{l}
\hat{x}_{1}=2 \int_{0}^{1}(2-3 \tau) y(t-\tau T) d \tau \\
\hat{x}_{2}=-\frac{6}{T} \int_{0}^{1}(1-2 \tau) y(t-\tau T) d \tau \\
\hat{x}_{3}=\frac{60}{T^{2}} \int_{0}^{1}\left(1-6 \tau+6 \tau^{2}\right) y(t-\tau T) d \tau
\end{array}\right.
$$


where $T$ represents the selected window, $y(t)$ is the signal and $\hat{x}_{1}, \hat{x}_{2}, \hat{x}_{3}$, respectively, represent the zero-, first-, and second-order derivative estimation.

This algebraic differentiator does not require any knowledge about the noise, and it provides explicit formula, and hence it is easy to be implemented in real-time applications. It is worth to notice that the integrals in formulas can be considered as low-pass filters, so high-frequency noises in the measurement signal can be attenuated by them. In addition, the estimates are obtained in a finite time $T$.

\subsubsection{High-Gain Differentiator}

Consider a single-input-single-output nonlinear system, having a uniform relative degree equal to the dimension of the state vector (A. Dabroom \& Khalil, 1997), then it can be transformed into normal form (Isidori, 2013):

$$
\begin{aligned}
& \dot{x}=A x+B[a(x) u+b(x)] \\
& y=C x
\end{aligned}
$$

where $(A, B, C)$ are canonical form matrices, and $a, b: \mathbb{R}^{n} \rightarrow \mathbb{R}$ are Lipschitz continuous functions. Let $u: \mathbb{R}_{+} \rightarrow \mathbb{R}$ be a bounded known input, then the observer equations take the following form:

$$
\dot{\hat{x}}=A \hat{x}+B\left[a_{0}(\hat{x}) \psi(\hat{x}, t)+b_{0}(\hat{x})\right]+H(y-C \hat{x})
$$

where $a_{0}(x)$ and $b_{0}(x)$ are nominal models of nonlinear functions $a(x)$ and $b(x)$, and $\mathrm{H}$ is the observer gain. It is shown in Esfandiari and Khalil (1992) that when the observer gain is chosen as

$$
H^{T}=\left[\begin{array}{llll}
\frac{\alpha_{1}}{\epsilon} & \frac{\alpha_{2}}{\epsilon^{2}} & \ldots & \frac{\alpha_{n}}{\epsilon^{n}}
\end{array}\right]
$$

then, the state reconstruction is achieved, where $\epsilon$ is a small positive parameter, and the positive constants $\alpha_{i}$ are chosen to make the roots of

$$
s^{n}+\alpha_{1} s^{n-1}+\cdots+\alpha_{n-1} s+\alpha_{n}=0
$$

having negative real parts (see A. M. Dabroom \& Khalil, 1999). The choice of $H$ sets the eigenvalues of $(A-H C)$ at $1 / \epsilon$ times the roots of (16). According to Esfandiari and Khalil (1992), the estimation error will decay to $O(\epsilon)$ after a short transient period.

\subsubsection{HOSM Differentiator}

The HOSM differentiator is proposed by Levant (2003). Let input signal $f(t)$ be consisting of a bounded Lebesgue-measurable noise with unknown features, and a unknown base signal $f_{0}(t)$ with the $n$-th derivative having a known Lipschitz constant 
$L>0$. Then, a recursive scheme can be used to get the $n$-th-order differentiator:

$$
\begin{aligned}
\dot{z}_{0} & =v_{0}, \quad v_{0}=-\lambda_{0}\left|z_{0}-f(t)\right|^{n /(n+1)} \operatorname{sign}\left(z_{0}-f(t)\right)+z_{1}, \\
\dot{z}_{1} & =v_{1}, \quad v_{1}=-\lambda_{1}\left|z_{1}-v_{0}\right|^{(n-1) / n} \operatorname{sign}\left(z_{1}-v_{0}\right)+z_{2}, \\
\vdots & \\
\dot{z}_{n-1} & =v_{n-1}, \quad v_{n-1}=-\lambda_{n-1}\left|z_{n-1}-v_{n-2}\right|^{1 / 2} \operatorname{sign}\left(z_{n-1}-v_{n-2}\right)+z_{n} \\
\dot{z}_{n} & =-\lambda_{n} \operatorname{sign}\left(z_{n}-v_{n-1}\right)
\end{aligned}
$$

Note that for $l<k$, the $k$-th-order differentiator provides for a better accuracy of $l$-th order derivative, than the $l$-th-order differentiator. So, in this paper, a secondorder differentiator is used to estimate the first-order derivative of blimp's altitude as proposed by Levant (2003), for input $f$ with $|\dddot{f}| \leq L$ :

$$
\begin{aligned}
& \dot{z}_{0}=v_{0}, \quad v_{0}=-3 L^{1 / 3}\left|z_{0}-f\right|^{2 / 3} \operatorname{sign}\left(z_{0}-f\right)+z_{1} \\
& \dot{z}_{1}=v_{1}, \quad v_{1}=-1.5 L^{1 / 2}\left|z_{1}-v_{0}\right|^{1 / 2} \operatorname{sign}\left(z_{1}-v_{0}\right)+z_{2} \\
& \dot{z}_{2}=-1.1 L \operatorname{sign}\left(z_{2}-v_{1}\right)
\end{aligned}
$$

\subsubsection{HOMD Differentiator}

Consider a nonlinear system of the form:

$$
\begin{aligned}
& \dot{\xi}=\eta(\xi, u) \\
& y=h(\xi)
\end{aligned}
$$

where $\xi$ is the state, $u$ is the control input which is sufficiently smooth, and $y$ is the output. Assume that (19) is locally observable and there exists a local state coordinate transformation and an output coordinate transformation which transform the nonlinear system 19 into the following canonical form:

$$
\begin{aligned}
\dot{x}_{1} & =x_{2}+f_{1}\left(x_{1}, u, \dot{u}, \ldots, u^{(r)}\right) \\
\dot{x}_{2} & =x_{3}+f_{2}\left(x_{1}, u, \dot{u}, \ldots, u^{(r)}\right) \\
\vdots & \\
\dot{x}_{n} & =f_{n}\left(x_{1}, u, \dot{u}, \ldots, u^{(r)}\right) \\
y & =x_{1}
\end{aligned}
$$

where $x \in \mathbb{R}^{n}$ is the new state and $r \in \mathbb{N}$. The observer is designed as follows:

$$
\begin{aligned}
& \frac{d \hat{x}_{1}}{d t}=\hat{x}_{2}+f_{1}\left(x_{1}, u, \dot{u}, \ldots, u^{(r)}\right)-\chi_{1}\left(x_{1}-\hat{x}_{1}\right) \\
& \frac{d \hat{x}_{2}}{d t}=\hat{x}_{3}+f_{2}\left(x_{1}, u, \dot{u}, \ldots, u^{(r)}\right)-\chi_{2}\left(x_{1}-\hat{x}_{1}\right) \\
& \quad \vdots \\
& \frac{d \hat{x}_{n}}{d t}=f_{n}\left(x_{1}, u, \dot{u}, \ldots, u^{(r)}\right)-\chi_{n}\left(x_{1}-\hat{x}_{1}\right)
\end{aligned}
$$


where the functions $\chi_{i}$ will be defined in such a way that the observation error $e=x-\hat{x}$ tends to zeros in finite time (see Perruquetti, Floquet, \& Moulay, 2008):

$$
\chi_{i}\left(e_{1}\right)=-k_{i}\left\lceil e_{1}\right\rfloor^{\alpha_{i}}
$$

where for any real number $x \in \mathbb{R}$ :

$$
\lceil x\rfloor^{\alpha}=|x|^{\alpha} \operatorname{sign}(x)
$$

and the parameters $\alpha_{i}$ can be chosen as

$$
\begin{aligned}
\alpha_{1} & =\alpha \in\left(\frac{n-1}{n}, 1\right), \\
\alpha_{2} & =2 \alpha-1, \\
\alpha_{3} & =3 \alpha-2, \\
\vdots & \\
\alpha_{n} & =n \alpha-(n-1)
\end{aligned}
$$

Then, the error dynamics of observer (21) become

$$
\begin{aligned}
& \dot{e}_{1}=e_{2}-k_{1}\left\lceil e_{1}\right\rfloor^{\alpha} \\
& \dot{e}_{2}=e_{3}-k_{2}\left\lceil e_{1}\right\rfloor^{2 \alpha-1} \\
& \quad \vdots \\
& \dot{e}_{n}=-k_{n}\left\lceil e_{1}\right\rfloor^{n \alpha-(n-1)}
\end{aligned}
$$

Consider a dilation with weights $\left(r_{1}, r_{2}, \ldots, r_{n}\right)=\left(1, \alpha_{1}, \ldots, \alpha_{n-1}\right)$, system $(25)$ is homogeneous of degree $d=\alpha-1$ with respect to these weights(Bernuau, Efimov, Perruquetti, \& Polyakov, 2014). In order to prove stability and convergence of this differentiator the following Lyapunov function can be used:

$$
\begin{aligned}
V_{\alpha}(e) & =\sigma^{T} P \sigma \\
\sigma & =\left[\begin{array}{llll}
\left\lceil e_{1}\right\rfloor^{1 / r_{1}} & \left\lceil e_{2}\right\rfloor^{1 / r_{2}} & \ldots & \left\lceil e_{n}\right\rfloor^{1 / r_{n}}
\end{array}\right]^{T} \\
& =\left[\begin{array}{llll}
e_{1} & \left\lceil e_{2}\right\rfloor^{1 / \alpha} & \ldots & \left\lceil e_{n}\right\rfloor^{1 /((n-1) \alpha-(n-2))}
\end{array}\right]^{T}
\end{aligned}
$$

where $P$ is the solution of the Lyapunov equation:

$$
\begin{aligned}
A_{0}^{T} P+P A_{0} & =-I \\
A_{0} & =\left(\begin{array}{cccc}
-k_{1} & 1 & 0 & 0 \\
-k_{2} & 0 & \ddots & 0 \\
\vdots & \vdots & \ddots & 1 \\
-k_{n} & 0 & \ldots & 0
\end{array}\right)
\end{aligned}
$$

and the gains $k_{i}$ are chosen such that $A_{0}$ is Hurwitz. Then, there exists $\epsilon \in\left[1-\frac{1}{n-1}, 1\right)$, such that, for all $\alpha \in(1-\epsilon, 1)$, system (25) is globally finite-time stable (Perruquetti et al., 2008). 


\subsubsection{Differentiator Comparison}

The High-Gain, HOSM and HOMD differentiator can be written in a similar formulation according to Perruquetti and Floquet (2007). In our case, the second-order differentiator can be written as

$$
\begin{aligned}
& \dot{x}_{1}=-k_{1}\left\lceil x_{1}-y\right\rfloor^{\alpha}+x_{2} \\
& \dot{x}_{2}=-k_{2}\left\lceil x_{1}-y\right\rfloor^{2 \alpha-1}+x_{3} \\
& \dot{x}_{3}=-k_{3}\left\lceil x_{1}-y\right\rfloor^{3 \alpha-2}
\end{aligned}
$$

where $y$ represents the measurement, $x_{1}, x_{2}$, and $x_{3}$, respectively, represent the zero-, first-, and second-order derivative estimation.

The gains $k_{1}, k_{2}$ and $k_{3}$ are selected in order to ensure that the corresponding polynomial is Hurwitz, and:

- For High-Gain differentiator $\alpha=1$;

- For HOMD differentiator $\alpha \in\left(\frac{2}{3}, 1\right)$;

- For HOSM differentiator $\alpha=\frac{2}{3}$.

This formula is easy to be implemented, and the parameter of HOMD differentiator is chosen as $\alpha=\frac{5}{6}$ in tests. Step altitude control is applied to compare the performances of the four differentiators.

First, it is the comparison of zero-order derivative estimation result:
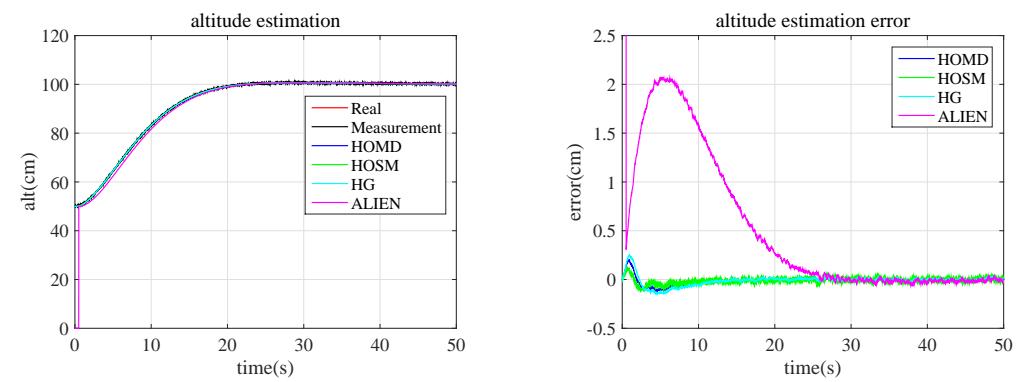

Figure 2. Altitude estimation comparison.

Then it is the comparison of first order derivative estimation result:
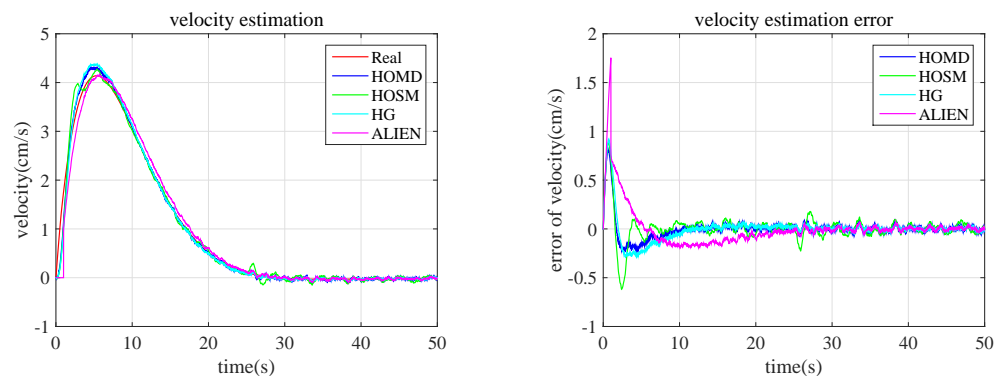

Figure 3. Velocity estimation comparison.

The 'Real' curve is generated using the model with parameters identified in Section 3.3 , and the sampling rate is $100 \mathrm{~Hz}$. In the 'Measurement' of altitude a white noise is added to simulate measuring error, which has a signal-to-noise ratio (SNR) of $25 \mathrm{~dB}$. 
From Fig. 2, it is clear that all the four differentiators can estimate the altitude signal after certain periods, but the ALIEN converges slower than the three others. This is due to the use of integrator which introduces delay of estimation in realtime applications. ALIEN differentiator can converge faster if the window $T$ of $(12)$ is smaller, but then it would be more sensitive to the noise. As the system studied in this paper has a time-varying delay which is non-ignorable, it is preferable to use a differentiator which converges faster.

As shown in Fig. 3, the HOMD, HOSM and High-Gain differentiator have similar performances. But it is worth to mention that HOSM has bigger fluctuation than the others after they converge, which means that HOSM is less robust to noises. HOMD has finite-time stability compared to High-Gain differentiator.

Therefore, in consideration of the convergence time and robustness to noises, the HOMD differentiator is used in this paper for $\dot{z}$ estimation. Then, the value of $\hat{\sigma}$ can be evaluated by the sign of $u$ and $\dot{z}$ using criteria:

$$
\begin{gathered}
\hat{X}=\left[\begin{array}{ll}
\hat{x_{1}} & \hat{x_{2}}
\end{array}\right]^{\mathrm{T}} \\
\hat{\sigma}= \begin{cases}1, & u \geq 0 \text { and } \hat{x_{2}} \geq 0 \\
2, & u \geq 0 \text { and } \hat{x_{2}}<0 \\
3, & u<0 \text { and } \hat{x_{2}} \geq 0 \\
4, & u<0 \text { and } \hat{x_{2}}<0\end{cases}
\end{gathered}
$$

\subsection{Predictor-based Controller Design}

Considering the system is time-delayed, a predictor-based controller is designed. It has two parts:

1) Predict state at time $t+\tau$ with Smith $(1959)$ predictor:

$$
\begin{aligned}
\hat{X}\left(t+\tau_{\text {nom }}\right)=e^{A_{\hat{\sigma}} \tau_{\text {nom }}} \hat{X}(t) & +\int_{-\tau_{\text {nom }}}^{0} e^{-A_{\hat{\sigma}} s} B_{\hat{\sigma}} u(t+s) \mathrm{d} s \\
& +\int_{-\tau_{\text {nom }}}^{0} e^{-A_{\hat{\sigma}} s} B_{\hat{\sigma}} \hat{d}\left(t+\tau_{\text {nom }}+s\right) \mathrm{d} s
\end{aligned}
$$

where $\hat{\sigma}$ is estimated according to $(29)$, and $\hat{d}$ is an estimate of the disturbance, which is described below.

2) Assign the controller output based on predictor result, disturbance term and desired state $X_{\text {set }}$ :

$$
u(t)=K_{\hat{\sigma}}\left(X_{\text {set }}-\hat{X}\left(t+\tau_{\text {nom }}\right)\right)-\hat{d}\left(t+\tau_{\text {nom }}\right) .
$$

From (30) and (31), it is clear that the disturbance term $d(t)$ has to be estimated in real time and predicted for the time interval $\left[t, t+\tau_{\text {nom }}\right]$. 


\subsection{Disturbance estimation and prediction}

The disturbance term in system description (6) represents the error between nominal model and blimp real situation.

In order to estimate the disturbance $d(t)$ in real time, a filter is designed:

$$
\left\{\begin{aligned}
\dot{X}_{\mathrm{fil}}(t) & =A_{\hat{\sigma}} X_{\mathrm{fil}}(t)+B_{\hat{\sigma}} u\left(t-\tau_{\mathrm{nom}}\right)+L\left(y(t)-y_{\mathrm{fil}}(t)\right) \\
y_{\mathrm{fil}}(t) & =C X_{\mathrm{fil}}(t)
\end{aligned}\right.
$$

where $L=\left[\begin{array}{ll}l_{1} & l_{2}\end{array}\right]^{\mathrm{T}}$ is the gain of filter. It is obvious that the filter has a similar form as a Luenberger observer for nominal model, i.e. (6) without disturbance term.

Let $e(t)=X(t)-X_{\text {fil }}(t)$ be the error between state vector of (6) and that of filter (32). Taking the time derivative of $e(t)$, we get

$$
\dot{e}(t)=\left(A_{\hat{\sigma}}-L C\right) e(t)+B_{\sigma} d(t)+\delta(t)
$$

where $\delta(t)=\left(A_{\sigma}-A_{\hat{\sigma}}\right) X(t)$. After simplification and assumption that $\sigma(t)=\hat{\sigma}(t)$, the expression of disturbance estimation is obtained:

$$
\hat{d}(t)=\frac{\ddot{e}_{1}(t)+\left(l_{1}-a_{\hat{\sigma}}\right) \dot{e}_{1}(t)+\left(l_{2}-a_{\hat{\sigma}} l_{1}\right) e_{1}(t)}{b_{\hat{\sigma}}}
$$

Note that $C=\left[\begin{array}{ll}1 & 0\end{array}\right]$, i.e. $y(t)=x_{1}(t)$, so $e_{1}$ is the difference between altitude measurement and filter output, and it is available for measurements. In order to assure the stability, the filter matrix $A_{\hat{\sigma}}-L C$ needs to be Hurwitz, and the gain $L$ can be chosen to make the filter converges five to six times faster than (6).

The calculation of $\dot{e}_{1}(t)$ and $\ddot{e}_{1}(t)$ is also realised by HOMD differentiator (28).

As it has been observed in experiments, the estimated disturbance signal is rather noisy; in order to decrease the chattering, we choose to use a time polynomial to fit $\hat{d}(t)$ in a sliding window, then the polynomial is used to predict $\hat{d}\left(t+\tau_{\text {nom }}\right)$.

\subsection{Determination of controller gain}

As system (6) is time-delayed with an uncertain bounded disturbance, a predictorbased controller (31) with disturbance compensation is designed. The problem remains to determine a gain of controller $K_{\hat{\sigma}}$ which can make the closed-loop system $\dot{X}(t)=$ $\left(A_{\sigma}-B_{\sigma} K_{\sigma}\right) X(t)$ stable.

Assume the gains of controller of switched system are chosen to be the same for all $\sigma$, i.e. $K_{\hat{\sigma}}=K$, to simplify calculation.

According to Liberzon and Morse (1999), if there exists a matrix $P$ and gain $K$, with $P=P^{\mathrm{T}}$, such that

$$
\left\{\begin{array}{l}
P \succ 0 \\
\left(A_{\sigma}-B_{\sigma} K\right)^{\mathrm{T}} P+P\left(A_{\sigma}-B_{\sigma} K\right) \prec 0, \forall \sigma \in \mathcal{P}
\end{array}\right.
$$

is satisfied, then the closed-loop switched system is stable. Transform (35) to 


$$
\left\{\begin{array}{l}
P^{-1} \succ 0 \\
P^{-1}\left(A_{\sigma}-B_{\sigma} K\right)^{\mathrm{T}}+\left(A_{\sigma}-B_{\sigma} K\right) P^{-1} \prec 0, \forall \sigma \in \mathcal{P}
\end{array}\right.
$$

Let $W=K P^{-1}$

$$
\left\{\begin{array}{l}
P^{-1} \succ 0 \\
P^{-1} A_{\sigma}^{\mathrm{T}}-W^{\mathrm{T}} B_{\sigma}^{\mathrm{T}}+A_{\sigma} P^{-1}-B_{\sigma} W \prec 0, \forall \sigma \in \mathcal{P}
\end{array}\right.
$$

where decision variables are $P^{-1}$ and $W$. If there exists solution for LMI (37), then the switched system (6) is globally uniformly exponentially stable.

A feasible solution of $P$ and $K$ can be solved by YALMIP toolbox of Matlab (Löfberg, 2004):

$$
\left\{\begin{array}{l}
P=\left[\begin{array}{ll}
0.2839 & 0.5822 \\
0.5822 & 3.2343
\end{array}\right] \\
K=\left[\begin{array}{ll}
0.6688 & 5.9713
\end{array}\right]
\end{array}\right.
$$

\section{Implementation on Real Blimp}

As it has been indicated in Section 3 , when implementing the designed altitude control algorithm on the real blimp, the OptiTrack camera system is used to improve the altitude measurement accuracy.

\subsection{OptiTrack-Enhanced Control System}

The enhanced control system scheme is shown in Fig. 4.

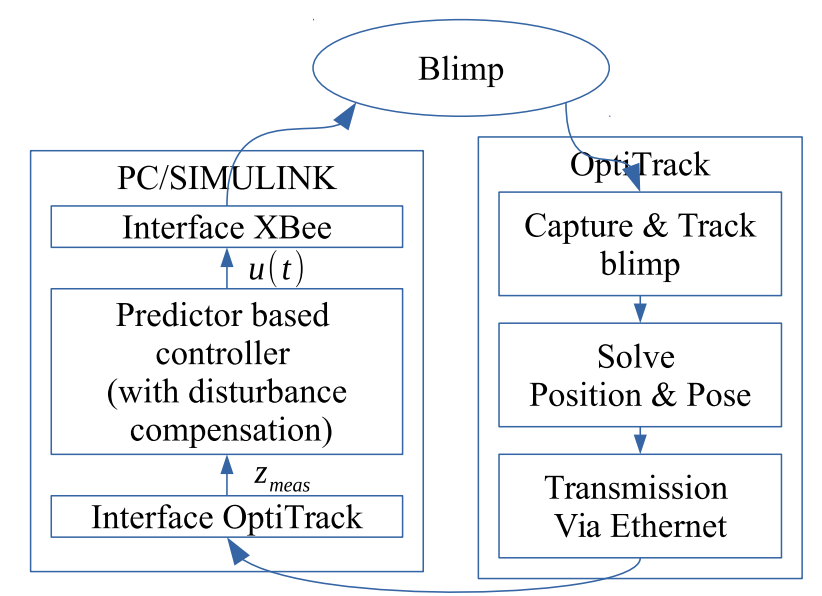

Figure 4. Scheme of Optitrack-enhanced blimp control system

According to this scheme, first, OptiTrack captures and tracks the blimp, then it reconstructs the position and pose of blimp (cameras are calibrated and reference frame is set beforehand in software) and sends message to PC where the controller is 
implemented in Matlab Simulink. In Simulink program, the differentiator in observer calculates $\dot{z}$ and observer gives estimation of switching signal $\hat{\sigma}$ (Section 4.1). Next, disturbance is estimated in real time by the filter (Section 4.3), then compensated in predictor-based controller (Section 4.2). Finally, the command of motors is sent to blimp control board via XBee wireless communication and the control loop is closed.

As presented in Section 4, the designed altitude controller is not complex; it has less than 100 lines of code, and it does not require high computational capacity to solve the command signal. Moreover, the size of program is less than $64 \mathrm{kB}$. Therefore, the Simulink program can be easily transformed to be used on embedded microprocessor.

\subsection{Results}

Experiments are made in a normal office room of height $3 \mathrm{~m}$. Five infrared cameras are installed on ground to form a circle, with their optic axis inclined upward to the vertical axis which passes the centre of the circle. The blimp floats inside the circle formed by the camera system, and when it moves to reach the desired altitude, it does not leave the view of cameras.

In the experiments, we have to adjust the additional weight for the blimp, in order to introduce disturbance for blimp system; the additional weight is set to make the blimp buoyancy force greater than its gravity, which means the blimp keeps rising if there is no command signal. A lightweight string has been attached to blimp control board to avoid the blimp from hitting the ceiling, and the string does not affect the blimp movement except when the blimp altitude exceeds the upper bound.

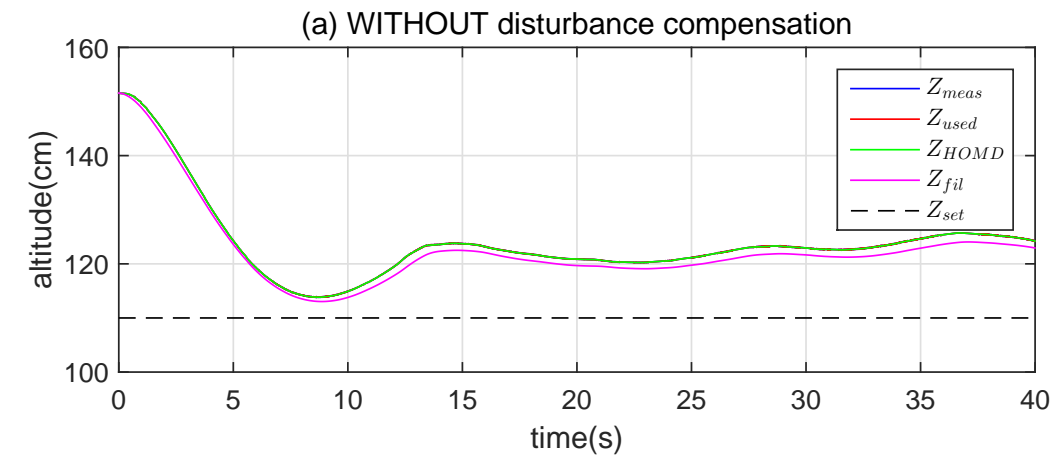

(b) WITH disturbance compensation

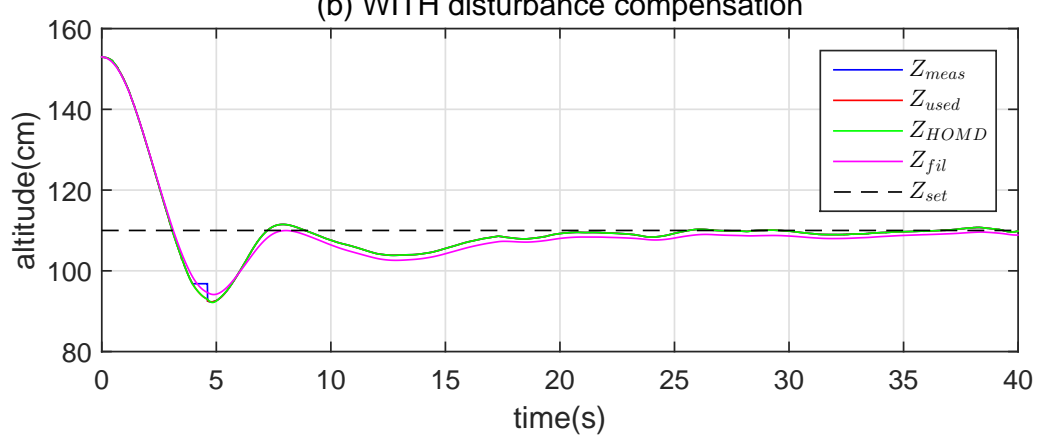

Figure 5. Blimp altitude controller performance comparison: (a) without disturbance compensation; (b) with disturbance compensation.

Fig. 5(a) shows the result of blimp altitude control without disturbance compensa- 
tion. It is obvious that the blimp altitude is oscillating, and it fails to reach the desired altitude.

Fig. 5(b) shows the result of blimp altitude control with disturbance compensation, as the method described in this paper. The blimp reaches the desired altitude with an error less than $\pm 5 \mathrm{~cm}$ within $10 \mathrm{~s}$.

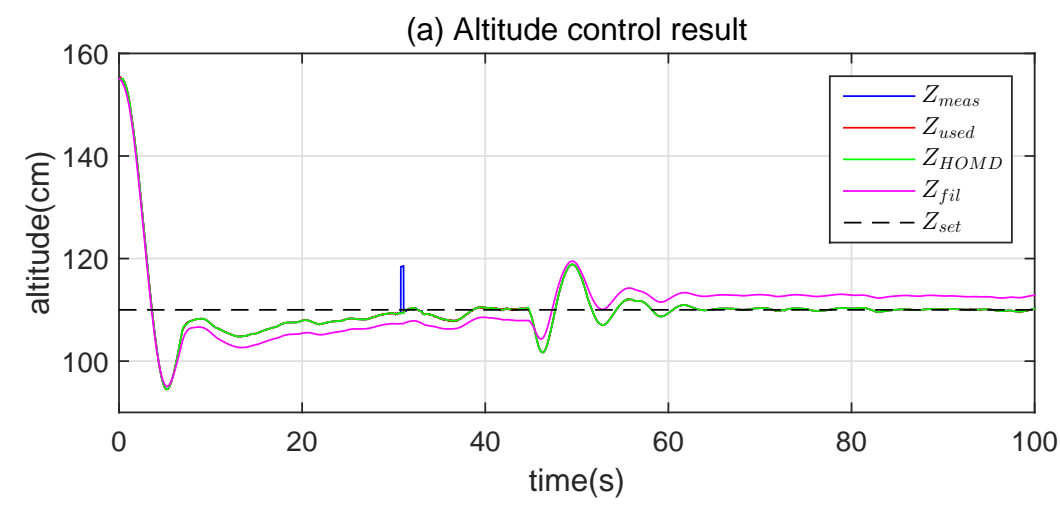

(b) Disturbance estimation result

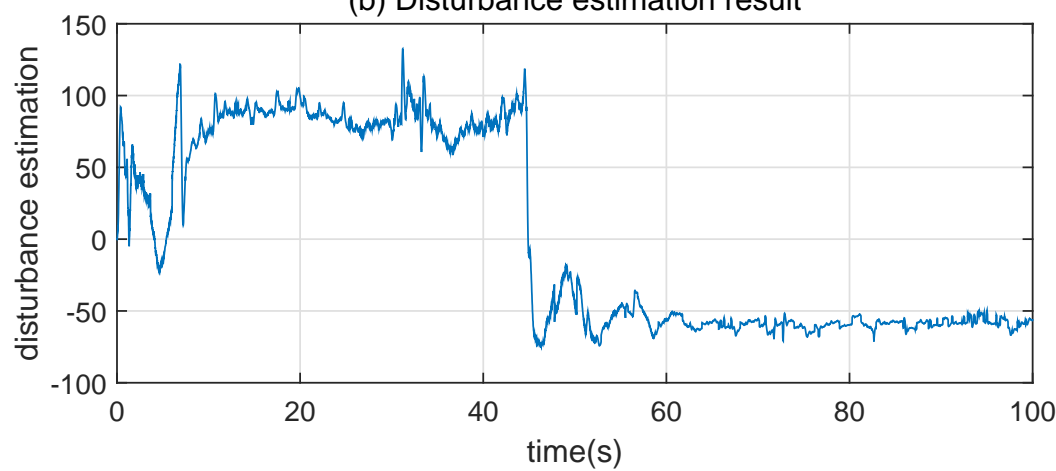

Figure 6. Blimp altitude control WITH disturbance compensation and additional weight change at 44.6s

Fig. 6(a) shows another test in which we add some additional weight to the blimp at $44.6 \mathrm{~s}$ to make the blimp gravity greater than buoyancy force. It is shown in the figure that the blimp altitude control is adapted to the disturbance change, and blimp returns to desired altitude with an error less than $\pm 5 \mathrm{~cm}$ within $7 \mathrm{~s}$.

Fig. 6(b) shows the disturbance estimation result $\hat{d}\left(t+\tau_{\text {nom }}\right)$. It is clear that $\hat{d}(t+$ $\tau_{\text {nom }}$ ) can reflect the disturbance caused by difference between blimp buoyancy force and gravity. Before the moment of 44.6s, the $c$ term in dynamical equation (3) is positive, and then it changes the sign, as with the disturbance estimation result.

Experiment results confirm that our designed altitude control method with disturbance compensation can successfully deal with the error between nominal model and blimp real situation. It improves the performance of blimp altitude controller.

A video of experimental result can be found here: https://drive.google.com/ drive/folders/1YmksewRWOodrUsALI--CyPM4qWGIUWv6?usp=sharing. 


\section{Conclusion and Future Work}

In this paper, the study of indoor small blimp robot's altitude control is presented. The work started from the modelling of indoor blimp robot, presenting an efficient way to identify the model's parameters. The model is identified as a switched system with a constant time-delay complemented with uncertain bounded disturbances. Next, in order to achieve full state estimation, HOMD, HOSM, High-Gain and ALIEN differentiators are discussed and compared, and HOMD differentiator is finally used to evaluate the blimp's vertical velocity. Then, a predictor-based controller is designed for altitude stabilising task. In order to compensate the disturbance in the model, a method to estimate and predict the disturbance is proposed with the help of HOMD differentiator again. When it comes to realisation on real blimp, the high-precision motion capture system is combined with the blimp system to improve measurement accuracy. Finally, we got a satisfying result in real tests, and demonstrated the performance of disturbance compensation of our controller in blimp altitude stabilising task.

The altitude control of indoor small blimp robot is just the first step of our final objective. In the future, we will continue the study on blimp robot, develop useful applications like long-term monitoring, or unknown environment exploring. To achieve that, we have to make the blimp system more autonomous, realise accurate position and pose estimation with information only coming from inside the system, and make the blimp more robust to disturbances.

\section{Acknowledgements}

The PhD student is sponsored by China Scholarship Council (CSC). Thanks to our team's engineer Mr. Xin Jin for his work on the development of blimp's first prototype. Thanks to Paul Lesur for his work on the communication interface between Simulink and OptiTrack and his help during tests.

\section{References}

Bernuau, E., Efimov, D., Perruquetti, W., \& Polyakov, A. (2014). On homogeneity and its application in sliding mode control. Journal of the Franklin Institute, 351 (4), 1866-1901.

Dabroom, A., \& Khalil, H. K. (1997). Numerical differentiation using high-gain observers. In Decision and control, 199\%., proceedings of the 36th ieee conference on (Vol. 5, pp. 47904795).

Dabroom, A. M., \& Khalil, H. K. (1999). Discrete-time implementation of high-gain observers for numerical differentiation. International Journal of Control, 72(17), 1523-1537.

Esfandiari, F., \& Khalil, H. K. (1992). Output feedback stabilization of fully linearizable systems. International Journal of control, 56(5), 1007-1037.

Fliess, M., \& Sira-Ramírez, H. (2003). An algebraic framework for linear identification. ESAIM: Control, Optimisation and Calculus of Variations, 9, 151-168.

Fukao, T., Fujitani, K., \& Kanade, T. (2003, Dec). Image-based tracking control of a blimp. In Decision and control, 2003. proceedings. 42nd ieee conference on (Vol. 5, p. 5414-5419 Vol.5).

Green, W. E., Sevcik, K. W., \& Oh, P. Y. (2005, July). A competition to identify key challenges for unmanned aerial robots in near-earth environments. In Icar '05. proceedings., 12th international conference on advanced robotics, 2005. (p. 309-315). 
Guerra, M., Vázquez, C., Efimov, D., Zheng, G., Freidovich, L., \& Perruquetti, W. (2015). $\varepsilon$-invariant output stabilization: Homogeneous approach and dead zone compensation. In Decision and control (cdc), 2015 ieee 54th annual conference on (pp. 6874-6879).

Hygounenc, E., Jung, I.-K., Soueres, P., \& Lacroix, S. (2004). The autonomous blimp project of laas-cnrs: Achievements in flight control and terrain mapping. The International Journal of Robotics Research, $23(4-5), 473-511$.

Isidori, A. (2013). Nonlinear control systems. Springer Science \& Business Media.

Kantor, G., Wettergreen, D., Ostrowski, J. P., \& Singh, S. (2001). Collection of environmental data from an airship platform. In Intelligent systems and advanced manufacturing (pp. 76-83).

Ko, J., Klein, D. J., Fox, D., \& Haehnel, D. (2007, April). Gaussian processes and reinforcement learning for identification and control of an autonomous blimp. In Proceedings 2007 ieee international conference on robotics and automation (p. 742-747).

Levant, A. (2003). Higher-order sliding modes, differentiation and output-feedback control. International journal of Control, 76 (9-10), 924-941.

Li, Y., Nahon, M., \& Sharf, I. (2011). Airship dynamics modeling: A literature review. Progress in Aerospace Sciences, $47(3), 217-239$.

Liberzon, D., \& Morse, A. S. (1999). Basic problems in stability and design of switched systems. IEEE Control systems, 19(5), 59-70.

Löfberg, J. (2004). Yalmip : A toolbox for modeling and optimization in matlab. In In proceedings of the cacsd conference. Taipei, Taiwan.

López Fernández, J., González, P., Sanz, R., \& Burgard, W. (2009). Developing a low-cost autonomous indoor blimp. Journal of Physical Agents, 3(1), 43-52.

Mboup, M., Join, C., \& Fliess, M. (2007, June). A revised look at numerical differentiation with an application to nonlinear feedback control. In 2007 mediterranean conference on control automation (p. 1-6).

Perruquetti, W., \& Floquet, T. (2007, Dec). Homogeneous finite time observer for nonlinear systems with linearizable error dynamics. In 200746 th ieee conference on decision and control (p. 390-395).

Perruquetti, W., Floquet, T., \& Moulay, E. (2008). Finite-time observers: application to secure communication. IEEE Transactions on Automatic Control, 53(1), 356-360.

Rao, J., Gong, Z., Luo, J., \& Xie, S. (2005). A flight control and navigation system of a small size unmanned airship. In Ieee international conference mechatronics and automation, 2005 (Vol. 3, p. 1491-1496 Vol. 3).

Rottmann, A., Zitterell, T., Burgard, W., Reindl, L., \& Scholl, C. (2007). Towards an experimental autonomous blimp platform. In the european conf. on mobile robots (ecmr). Citeseer.

Seber, G. A. F., \& Wild, C. J. (2003). Nonlinear regression (Hoboken, Ed.). WileyInterscience.

Sira-Ramirez, H., \& Fliess, M. (2006). An algebraic state estimation approach for the recovery of chaotically encrypted messages. International Journal of Bifurcation and Chaos, 16(02), 295-309.

Smith, O. J. (1959). A controller to overcome dead time. iSA journal, 6(2), 28-33.

Wyeth, G., \& Barron, I. (1998). An autonomous blimp. In Field and service robotics (pp. 464-470).

Zufferey, J.-C., Guanella, A., Beyeler, A., \& Floreano, D. (2006). Flying over the reality gap: From simulated to real indoor airships. Autonomous Robots, 21 (3), 243-254. 